CYBERNETICS AND INFORMATION TECHNOLOGIES • Volume 16, No 6

Special issue with selection of extended papers from 6th International Conference on Logistic, Informatics and Service Science LISS'2016

Sofia $\bullet 2016$

\title{
A Fast and Simple Adaptive Bionic Wavelet Transform: ECG Baseline Shift Correction
}

\author{
Ludi Wang, Xiaoguang Zhou, Ying Xing, Siqi Liang \\ Automation School, Beijing University of Post and Telecommunications, Beijing 100876, China \\ Emails: wld@bupt.edu.cn zxg@bupt.edu.cn xingying@bupt.edu.cn 13261712736@163.com
}

\begin{abstract}
An ECG baseline shift correction method is presented on the base of the adaptive bionic wavelet transform. After modifying the bionic wavelet transform according to the characteristics of the ECG signal, we propose a novel adaptive BWT algorithm. Using the contaminated and actual data in the MIT-BIH database, the method of fast and simple adaptive bionic wavelet transform can effectively correct the baseline shift under the premise of maintaining the geometric characteristics of the ECG signal. Evaluation of the proposed method shows that the average improvement SNR of FABWT is $2.187 \mathrm{~dB}$ more than the CWT-based best case result.
\end{abstract}

Keywords: Baseline shift, Bionic Wavelet Transform (BWT), fast algorithm, MIT-BIH.

\section{Introduction}

ElectroCardioGraphy (ECG) can record the electrical activity of heart over a period of time when electrodes are placed on the skin. For the diagnosis of heart disease, the electrocardiogram (ECG) is seen as the most important non-invasive tool. Thanks to the development of IOT, people can easily obtain the digital format of the signal with intelligent hardware. But the noise generated in the process of data gathering can make heavy interference in the latter process. There are many kinds of noise [1] when an electrocardiogram is recorded, such as baseline wandering, power-line interference, etc.

Since the intelligent diagnosis is mostly based on the pure measurements, the extraction from noisy measurements has been one of the most significant jobs in the field of biomedical signal processing and the intelligent health hardware. The aim of 
this paper is to remove baseline wandering caused by low pass noise as shown on Fig. 1.

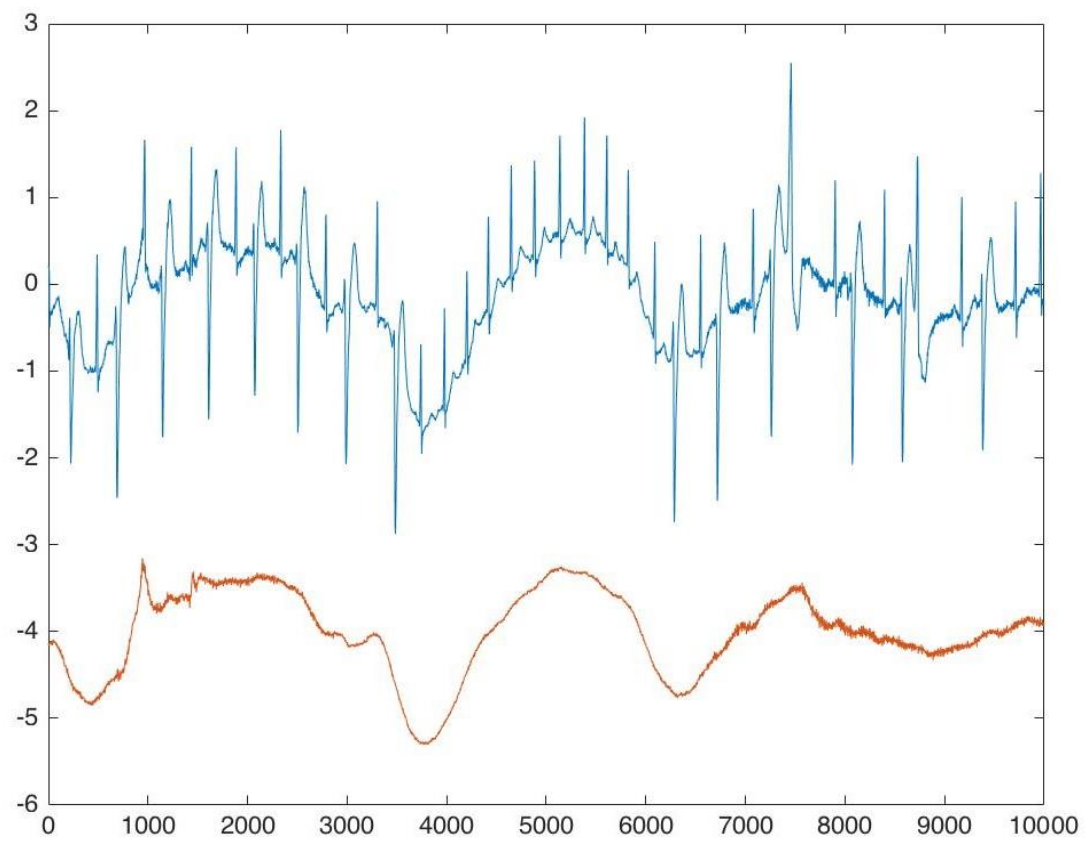

Fig. 1. Baseline wandered ECG with its baseline trace

Some methods have been proposed to remove baseline wandering in the past years. One of the common approaches is the adaptive filters architecture [2], but the method loses high-frequency data and so causes linear anamorphosis. Cliff ord, A zu aje and $\mathrm{McSh}$ arr y [3] proposed a statistical technique based on Principal Component Analysis (PCA), but the method is based on the accurate segmentation of ECG waveform so cause the timeliness reduces. Over past several years, Wavelet Transform (WT) [4-7] methods have received great deal of attention for signals denoising in biomedicine field. Baseline estimation using cubic spline [8], baseline construction by linearly interpolating between pre-known isoelectric levels estimated form PR intervals [9] are methods also used in this field. Besides the above algorithms, Yao and $\mathrm{Zhang}$ [10] introduced a new wavelet transform called Bionic Wavelet Transform (BWT). This method is mainly developed and being optimized by the human auditory system and received great results in speech processing [11-13].

However, as Yao says, this method is well applied to the process of speech signal with high frequency, but the frequency of ECG signal is lower. Also the decomposition and reconstruction of this method is too complicated. This motivated us to improve BWT with new modifications to be adjusted for ECG denoising, and also combine it with Flourier Transform (FT) in the complex field to simplify the decomposition and reconstruction, which has never been proposed in the past. 
The paper is organized as follows. Section 2 describes theoretical background and the definition of the bionic wavelet transform. In Section 3 we modify the BWT in the complex field and give the pseudo codes of some steps. Section 4 shows the results compared with CWT. Finally, the discussion and conclusion are given in Section 5 .

\section{Bionic wavelet transform}

The structure of the human ear is mainly composed of the external ear, middle ear and inner ear. The main function of external ear and middle ear is to enhance the external sound, while the basilar membrane in the inner ear of the cochlea is mainly responsible for the accepting and dividing frequency. Because the basilar membrane can change the resolution ability of the signal according to the frequency, it has better resolution of low frequency signal.

In previous work, a nonlinear auditory model by Giguere was adopted and modified as an OAE mode [14-16]. Based on the model, Y a o and $\mathrm{Zh}$ ang [10] replaced the constant Q0 of WT by a variable QT and proposed the bionic wavelet transform. To do this, we can make changes in the mother function of the wavelet transform. In the paper [10] the mother function is Morlet function; it is satisfied as the admissible condition for the mother wavelet. The oscillating $\varphi(t)$ can be represented as

The BWT is defined as

$$
\begin{gathered}
\varphi(t)=\tilde{\varphi}(t) \cos \omega_{0} t . \\
\tilde{\varphi}(t)=e^{-\left(\frac{t}{T_{0}}\right)^{2}} .
\end{gathered}
$$

$$
X_{\mathrm{BVT}}(\alpha, \tau)=\frac{1}{T(\alpha, \tau) \sqrt{\alpha}} \int x(t) \varphi^{*}\left(\frac{t-\tau}{T(\alpha, \tau) \alpha}\right) \times \exp \left(-j \omega_{0}\left(\frac{t-\tau}{\alpha}\right)\right) d t
$$

(4) $T(\alpha, \tau+\Delta \tau)=\left(1-G_{1} \frac{\mathrm{BWT}_{\mathrm{s}}}{\mathrm{BWT}_{\mathrm{s}}+\left|\mathrm{BWT}_{\mathrm{f}}(\alpha, \tau)\right|}\right)^{-1}\left(1+G_{2}\left|\frac{\partial \mathrm{BWT}_{\mathrm{f}}(\alpha, \tau)}{\partial t}\right|\right)^{-1}$.

According to the auditory model, $G_{1}=0.87, G_{2}=45$ respectively represents the active factors of the cochlea. $\mathrm{BWT}_{\mathrm{S}}=0.8$ is saturation constant in the auditory model. $\mathrm{BWT}_{\mathrm{f}}$ is the coefficient of the BWT at current time and scale.

In implementation, BWT coefficient can be easily calculated based on the character of Morlet function by and $K$ is the factor depending on $T$ as

$$
K=\frac{T_{0} \int_{-\infty}^{+\infty} e^{-t^{2}} d t}{\sqrt{T^{2}+1}}=\frac{1.7725 T_{0}}{\sqrt{T^{2}+1}} .
$$




\section{Fast and simple bionic wavelet transform optimization in the complex} field for ECG analysis

Although the character of BWT, it can process the low frequency signal and has received great result in the enhancement of speech. The decomposition and reconstruction can be complicated and may cause anamorphosis, so it motivated us to apply it with Fourier Transform in the complex field to resolve the problem.

The WT transform can be calculated by FT in each scale a and time $\tau$ shift as

$$
\mathrm{WT} f=\langle f(t), h(t)\rangle=F(w) * H(w) .
$$

We can change the differential equation into multiplication by FT and IFT as

$$
\begin{gathered}
f(t) \leftrightarrow F(\Omega) . \\
\frac{d f(t)}{d t} \leftrightarrow j \Omega F(\Omega) .
\end{gathered}
$$

So, in the BWT, we can calculate the $\frac{\partial \mathrm{BWT}_{f}(\alpha, \tau)}{\partial t}$ based on above theorem. After that we can calculate each scale and time shift WT coefficients with FT, and also the BWT coefficients. Fig. 2 shows the time-frequency representation for an ECG signal with both WT and BWT. The steps of our method are as shown below.
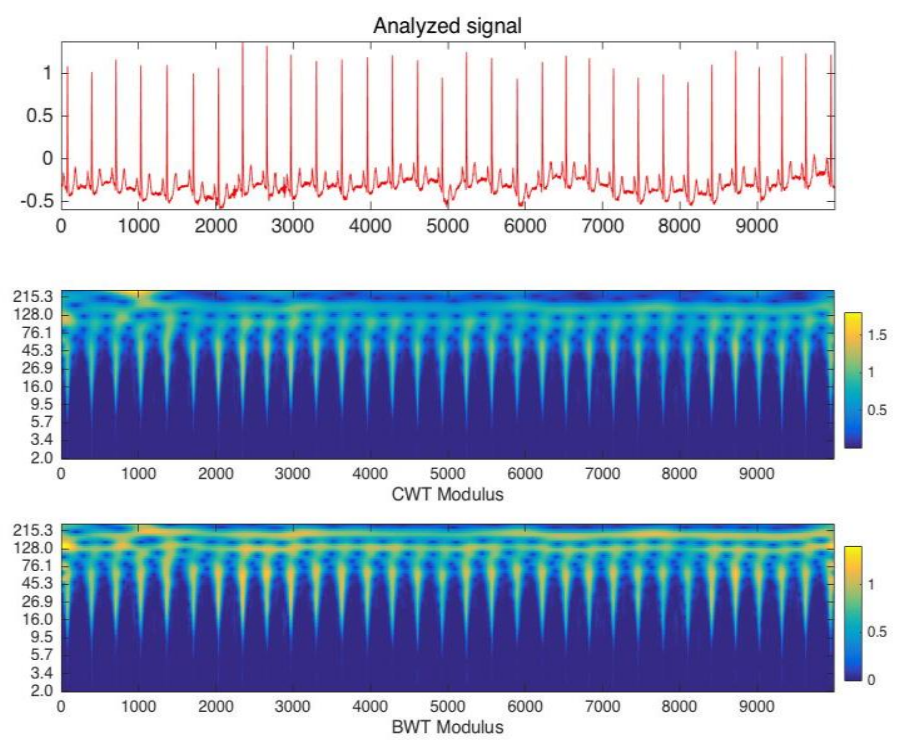

Fig. 2. Modulus for an ECG signal with both WT and BWT

\subsection{Transform the ECG signal by BWT}

Respectively transform the noisy ECG signal and mother function by Flourier Transform, then multiply them in each scale to obtain the coefficients of WT;

Calculate the BWT coefficients of noisy ECG signal based on the (5) and (6). The pseudo codes are given in Fig. 3. 


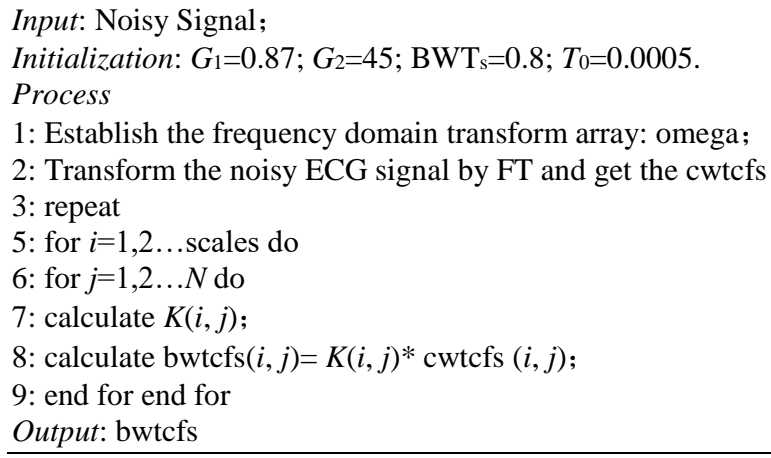

Fig. 3. Pseudo codes

\subsection{Denoising noisy ECG signal with threshold}

After BWT transform, we use the denoising method presented in Fig. 3 to reduce the amount of noise contamination. Here we use Donoho's approach for denoising $[17,18]$. Donoho proposed the formula based on white Gaussian noise assumption as follows:

$$
\lambda_{i}=\sigma_{i} \sqrt{2 \log _{2} N},
$$

where $\sigma_{i}$ is noisy level and estimated value is

$$
\sigma_{i}=\mathrm{MAD}_{i} / 0.674 \text {. }
$$

The $\mathrm{MAD}_{i}$ is the absolute median estimated on the $i$ scale of bionic wavelet coefficients.

Since the BWT is depending on the $T$-function, we consider the influence of $T$ and use a new threshold. Say a d, O mid and Shamsollahi [19] derived the threshold formula as

$$
\text { thr }=\frac{\sigma}{\sum_{i} a_{i} T_{\mathrm{fs}}(i)} \sqrt{2 \log _{2} N},
$$

where $a_{i}=1 / i$ and $T_{\mathrm{fs}}(i)$ is the mean of the first scale of $T$.

In our method, we do the threshold in every scale and modify the threshold as:

$$
\operatorname{thr}(i)=\frac{\sigma}{\sum_{i} b_{i} T(i)} \sqrt{2 \log _{2} N}
$$

So, it can reduce the noise in each scale and fit the original thought of Donoho's approach.

\subsection{Reconstruction of pure ECG signal}

In this step, we use the IFT to reconstruct the pure ECG signal. And also it is the step we correct the baseline wandering further by introduced the adaptive factor $q$.

Let $f_{0}$ be the initial center frequency of the mother wavelet. In [10], it is equal to $15165.4 \mathrm{~Hz}$ and as the scale changes, the center frequency will decrease as follows: 


$$
f_{w}=\frac{f_{0}}{q^{m}}, \quad q>1 .
$$

But the value of $f_{0}$ depends on the character of auditory signal, while using ECG we do not need such high $f_{0}$. Sayadi, Omid and Shamsollahi [19] has chosen $f_{0}=400$ after analyzing the character ECG signal. However, here we chosen the frequency of heartbeat as $f_{0}$ to reflect the difference of inter-ECGs (it means the ECG of different people).

Unlike [10] and [19], in our method $q$ is not a global constant or fixed value, but an adaptive constant based on scales. We defined the $q(i)$ equal to $1+T(i, 2)$ and received better result.

\section{Simulation and result}

In order to better verify our method, we used three types of ECG database for our experiment. We used the MIT-BIH arrhythmia database [20] and contaminated it with the baseline wandering noise database provided by Phsionet [21] as contaminated signal. For real signal, we used the noisy ECGs provided by Phsionet directly.

We have run the baseline wandering cancellation algorithm with both WT and FABWT. The results are provided in Figs 4 and 5.
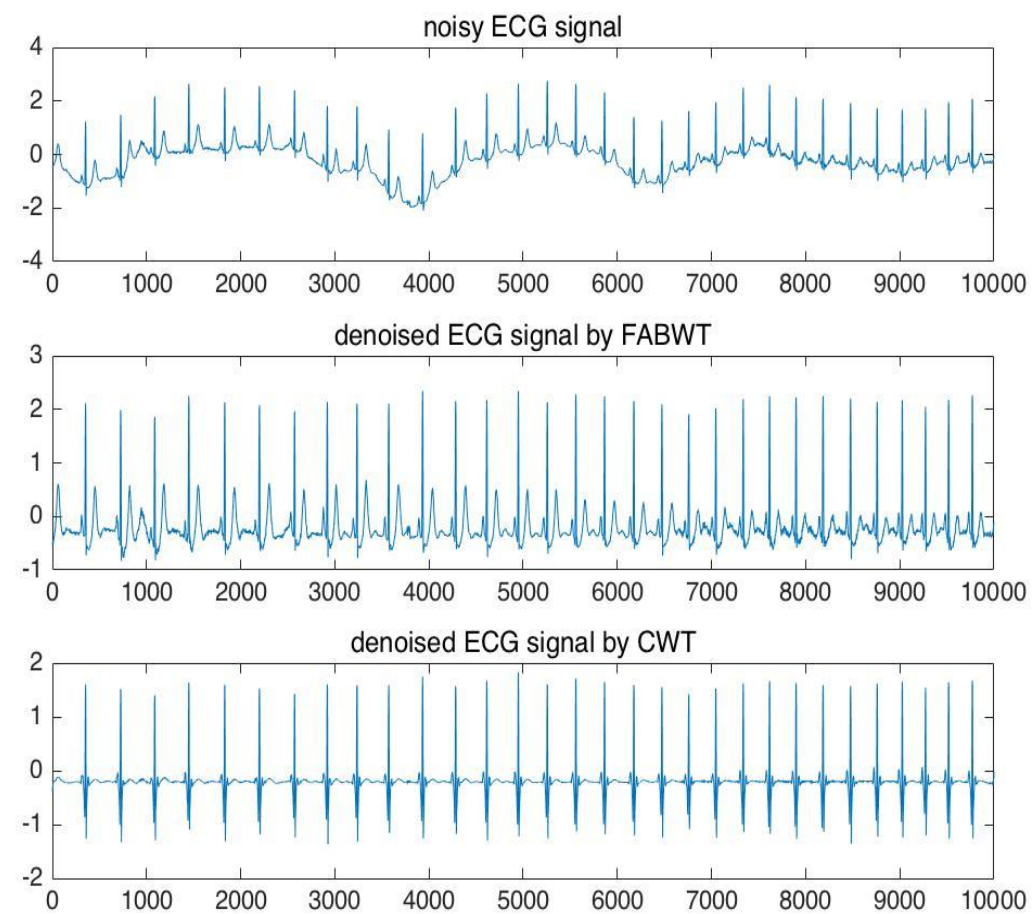

Fig. 4. Baseline wandering reduction based FABWT and CWT for the contaminated signal 

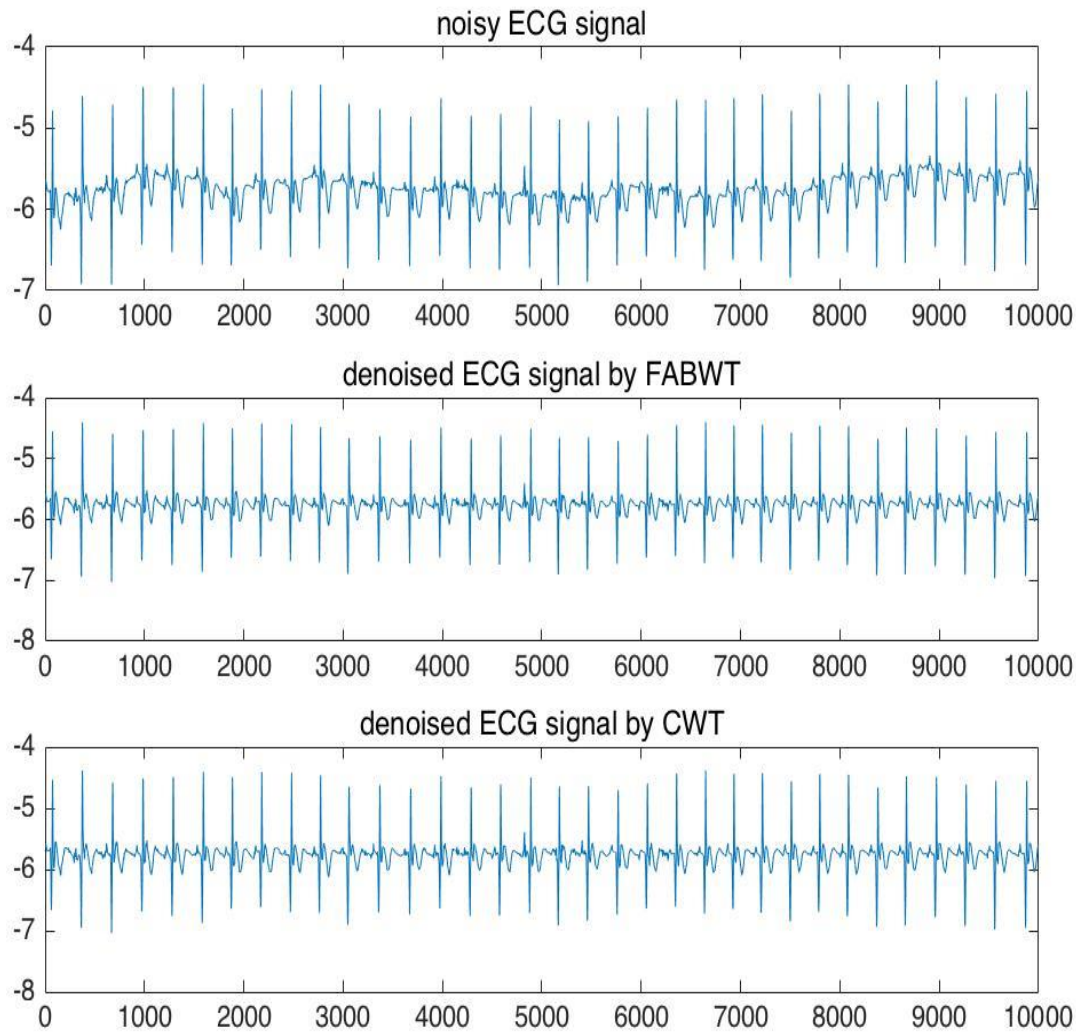

Fig. 5. Baseline wandering reduction based on FABWT and CWT for the real signal

The results show that the denoising method based on WT may cause a difference on the QRS complex and $T$ waves while FABWT method can maintain the morphology better.

For evaluating the performance of the proposed FAWBT, we have used the SNR improvement as the validation criteria. The SNR can be presented as

$$
\mathrm{SNR}=10 \log \left(\frac{\sum_{i}|x(i)|^{2}}{\sum_{i}\left|x_{d}(i)-x(i)\right|^{2}}\right) .
$$

The SNR improvement is

$$
\mathrm{SNR}_{\text {imp }}=\mathrm{SNR}_{\text {output }}-\mathrm{SNR}_{\text {input }}=10 \log \left(\frac{\sum_{i}\left|x_{\mathrm{n}}(i)-x(i)\right|^{2}}{\sum_{i}\left|x_{\mathrm{d}}(i)-x(i)\right|^{2}}\right),
$$

where $x$ presents the pure ECG signal, $x_{\mathrm{d}}$ presents the denoised ECG signal and $x_{\mathrm{n}}$ presents the noisy ECG signal. We can easily notice that the larger $\mathrm{SNR}_{\mathrm{imp}}$, the better denoise performance. For the experiment, we have chosen $N=10,000$ samples of the artificial contaminated ECG signal database. Table 1 shows the results of baseline 
wandering correction of contaminated ECG signal by Continuous Wavelet Transform (CWT) and our method.

Table 1. Results of baseline wandering correction based on CWT and FABWT

\begin{tabular}{|c|c|c|}
\hline \multirow{2}{*}{ Record No } & \multicolumn{2}{|c|}{ SNR $_{\text {imp }}, \mathrm{dB}$} \\
\cline { 2 - 3 } & CWT & FABWT \\
\hline 106 & 26.89 & 29.02 \\
\hline 108 & 27.5 & 28.5 \\
\hline 200 & 17.51 & 18.88 \\
\hline 202 & 12.7 & 14.6 \\
\hline 214 & 17.21 & 18.93 \\
\hline 228 & 12.52 & 17.52 \\
\hline Average & 19.055 & 21.242 \\
\hline
\end{tabular}

The average SNR improvement of FABWT is $2.187 \mathrm{~dB}$ more than the CWT-based results. The results provide supportive evidence to claim that BWT has some advantages over the CWT. Also, in contrast to CWT method which may cause some distortions in the features, the processed signal with FABWT can maintain the morphology of ECG signal.

\section{Discussion and conclusions}

We have applied the bionic wavelet transform to the baseline wandering reduction of ECG signal. In order to reduce the complexity of decomposition and reconstruction, we modify it in combination with FT in the complex field. Also, instead of setting $q$ a global constant or fixed value, we have used an adaptive factor. Moreover, we modify the traditional rules for threshold to adaptive ones based on the multiresolution scheme.

The great performance of FABWT is based on the multi-resolution analysis and multi-adaptive scheme. The method may provide a fast and simple algorithm for the problem of ECG denoising.

\section{References}

1. Clifford, G. D., F. A z u a je, P. M c Sh arry. Advanced Methods and Tools for ECG Data Analysis. Linear Filtering Methods. Chapter 5. Boston, Artech House Publishers, 2006.

2. Thakor, N. V., Y. S. Zhu. Applications of Adaptive Filtering to ECG Analysis: Noise Cancellation and Arrhythmia Detection. - IEEE Transactions on Biomedical Engineering, Vol. 38, 1991, No 8, pp. 785-794.

3. Clifford, G. D., F. A z u a je, P. M c Sharry. Advanced Methods and Tools for ECG Data Analysis. Artech House Publishers, 2006, pp. 164-167.

4. D a q r o u q, K., et al. ECG Baseline Wander Reduction Using Discrete Wavelet Transform. - Asian Journal of Information Technology, Vol. 4, 2005, No 11, pp. 989-995.

5. Ke st le r, H. A., M. Ha s ch ka, W. Krat z et al. Denoising of High-Resolution ECG Signals by Combining the Discrete Wavelet Transform with the Wiener Filter. - Proceedings of Computers in Cardiology, 1998, pp. 233-236.

6. Martín e z, J. P., et al. A Wavelet-Based ECG Delineator: Evaluation on Standard Database. IEEE Trans. Biomed. Eng., Vol. 51, 2004, No 4, pp. 570-581. 
7. S in gh, B. N., A. K. Ti w a ri. Optimal Selection of Wavelet Basis Function Applied to ECG Signal Denoising. - Digit. Signal Process, Vol. 3, 2006, No 16, pp. 275-287.

8. M e y e r, C. R., H. N. K e i s e r. Electrocardiogram Baseline Noise Estimation and Removal Using Cubic Splines and State-Space Computation Techniques. - Computers and Biomedical Research, Vol. 10, 1977, No 5, pp. 459-470.

9. M a c F arla n e, P. W., J. P e d e n, J. Le n nox, M. P. W at t s, T. D. Lawri e. The Glasgow System. Trends in Computer Processed Electrocardiograms. - In: Proc. of IFIP Working Conference on Trends in Computer-Processed Electrocardiograms, 1977, pp. 143-150.

10. Y a o, J., Y. T. Z h a n g. From Otoacoustic Emission Modeling to Bionic Wavelet Transform. In: Proc. of 22nd Annual International Conference of the IEEE Engineering in Medicine and Biology Society, Vol. 1, 2000, pp. 314-316.

11. Mourad, T., et al. Speech Enhancement Based on Stationary Bionic Wavelet Transform and Maximum a Posterior Estimator of Magnitude-Squared Spectrum. - International Journal of Speech Technology, 2016, pp. 1-14.

12. M o u r a d, T., et al. Bionic Wavelet Based Denoising Using Source Separation. - International Journal of Computers Communications \& Control, Vol. 7, 2012, No 3, pp. 529-539.

13. Z u o, D. G., et al. Research on a Speech Denoising Method Based on Bionic Wavelet Transform and Fuzzy Reasoning. - Electronic Design Engineering, Vol. 1, 2013, No 51.

14. K e m p, D. T., et al. Stimulated Acoustic Emissions from Within the Human Auditory System. J. Acoust. Soc. Amer., Vol. 64, 1978, No 5, pp. 1386-1391.

15. Pro b s t, R., et al. A Review of Otoacoustic Emissions. - J. Acoust. Soc. Amer., Vol. 89, 1991, No 5, pp. 2027-2066.

16. L i m, D. J., F. K a 1 i n e c. Cell and Molecular Basis of Hearing. - Kidney Int. Suppl., Vol. 53, 1998, pp. 104-113.

17. D o n o h o, D. L., et al. De-Noising by Soft-Thresholding. - IEEE Transactions on Information Theory, Vol. 41, 1995, No 3, pp. 613-627.

18. D o n o h o, D. L., I. M. J o h n s t o n e. Adapting to Unknown Smoothness via Wavelet Shrinkage. - Journal of the American Statistical Association, Vol. 90, 1995, No 432, pp. 1200-1224.

19. S a y a di, O., M. B. S h a m s o 11 a hi. Multiadaptive Bionic Wavelet Transform: Application to ECG Denoising and Baseline Wandering Reduction. - Eurasip Journal on Advances in Signal Processing, Vol. 1, 2007, pp. 1-11.

20. The MIT-BIH Arrhythmia Database. https://physionet.org/physiobank/database/mitdb/

21. The MIT-BIH Noise Stress Test Database. https://physionet.org/physiobank/database/nstdb/ 